\title{
RSPO Gene Fusion Positive
}

National Cancer Institute

\section{Source}

National Cancer Institute. RSPO Gene Fusion Positive. NCI Thesaurus. Code C153271.

Indicates that the expression of a fusion containing RSPO family gene sequences has been detected in a sample. 